COLLOQUIUM MATHEMATICUM

\begin{tabular}{lll}
\hline VOL. VI DÉDIÉ À M. CASIMIR KURATOWSKI & 1958 \\
\hline
\end{tabular}

\title{
О ПРОДОЛЖЕНИИ ЛОКАЛЬНО КОНЕЧНЫХ ПОКРЫТИЙ
}

M. КА TE TOB (ПРАГA)

На̇стоящая заметка имеет в значительной мере обзорный характер. Многие содержащиеся в ней результаты уже указаны в литературе или легко выводятся из уже известных теорем; однако, возможно, не лишено интереса их изложение вместе с некоторыми, быть может, новыми соображениями. В связи с указанным, доказательства значительно сокращены; очевидные доказательства (или части доказательств) опускаются.

1. Если каждому элементу $\alpha$ некоторого непустого „множества индексов" $A$, сопоставлен элемент (обычно, множество) $X_{a}$, то мы говорим, что дана система $\left\{X_{a} ; \alpha \in A\right\}$ или, короче, $\left\{X_{a}\right\}$; множество всех конечных $a \subset A$ будем обозначать через [A]. Пусть имеются системы множеств $\left\{X_{\alpha} ; \alpha \epsilon A\right\},\left\{Y_{\beta} ; \beta \epsilon B\right\}$. Если для каждого $\alpha$ имеем $X_{\alpha} \subset Y_{\beta}$ при подходящем $\beta$, то $\left\{X_{a}\right\}$ вписана в $\left\{Y_{\beta}\right\}$. При $A=B$ называем $\left\{X_{\alpha}\right\},\left\{Y_{\beta}\right\}$ комбинаторно подобньми и пишем $\left\{X_{\alpha}\right\} \cong\left\{Y_{a}\right\}$, если для любого $a \epsilon[A]$ множества $\cap X_{\alpha}, \cap Y_{\alpha}$ или оба равны ø, или оба непусты. Если для всякого $\beta$ имеем $X_{\alpha} \cap Y_{\beta}=\varnothing$ только для конечного числа индексов $\alpha$, то $\left\{X_{\alpha}\right\}$ конечна относительно $\left\{Y_{\beta}\right\}$. Если $\left\{X_{a}\right\}$ конечна относительно $\left\{X_{a}\right\}$, то $\left\{X_{a}\right\}$ звездно конечна. Кратностью $\left\{X_{a}\right\}$ называется или $\infty$, или же, если оно существует, наименьшее $n=0,1,2, \ldots$ такое, что $\bigcap X_{a}=\varnothing$ для $a \subset A$, содержащих более $n$ элементов.

Пространством мы называем топологическое пространство, удовлетворяющее аксиомам I-III из [6]. В дальнейшем буква $P$ всегда обозначает пространство, буквы (часто с индексами) $F, G, H, S, U$, $V, X$ - его подмножества (подпространства). Система $\left\{X_{\alpha}\right\}$ называется покрытием подпространства $S \subset P$, если $\cup X_{\alpha}=S\left({ }^{1}\right)$, притом открытым (замкнутым), если $X_{a}$ открыты (замкнуты) в $S$, локально конечным, если $\left\{X_{a}\right\}$ локально конечна в $S$; система $\left\{X_{a}\right\}$ называется

(1) При знаке соединения мы, как правило, опускаем индексы, так как обычно ясно по какому множеству индексов оно берется.

Colloquium Mathematicum vi 
локально конечной в $S$, если $X_{a} \subset S$ и существет открытое покрытие $S$, относительно которого $\left\{X_{a}\right\}$ конечна. Если $S=P$, то мы обычно говорим грэсто о локально конечной системе, открытом покрытии и т. п. Общеизвестные определения и теоремы, применяемые в дальнейшем, мы не будем напоминать; их можнно найти напр. в [4] и [6]. Отметим только, что $P$ называется счетно паракомпактным, если в каждое его счетное открытое покрытие можно вписать локально конечное открытое покрытие.

Напомним теперь несколько теорем (доказательство 1.1 не представляет труда; доказательство 1.2 содержится напр. в [1]; $1.3-$ в [8]; $1.4-$ в [3] (см. такभе [5]); $1.5-$ в [7]).

1.1. Eсли $\left\{X_{a} ; a \in A\right\}$ локально конечна в $S \subset P, \operatorname{mo}\left\{\bigcap_{a \in a} X_{\alpha} ; a \in[, A]\right\}$ такжсе локально конечна в $S$.

1.2. Если $P$ нормально, то для любого локально конечного открытого покрытия $\left\{G_{a}\right\}$ существуют замкнутые $F_{a} \subset G_{a}$ maкие, что $\bigcup F_{a}=$ $=P$.

1.3. Если $P$ нормально, $F_{n} \subset U_{n} \subset P, \quad n=1,2, \ldots, \cup F_{n}=P$, $F_{n}$ замкнуты, $U_{n}$ открыты, то в $\left\{U_{n}\right\}$ мохсно вписать счетное звездно конечное открытое покрытие.

1.4. Если $P$ нормально, $F_{k} \subset P$ замкнуты, $k=1,2, \ldots,\left\{F_{k c}\right\}$ локально конечна в $\bigcup F_{k}$, то существуют открытые $G_{k} \supset F_{k}$ maкuе, что $\left\{G_{k}\right\} \cong\left\{F_{k}\right\}$.

1.5. Для того, чтобы нормальное $Р$ было счетно паракомпактным, необходимо и достаточно кажсде из следующих условий: (а) в любое счетное открытое покрытие мохсно вписать счетное звездно конечное открытое покрытие; (b) если $U_{n} \subset P$ oткрытыл, $\bigcup_{n=1}^{\infty} U_{n}=P$, mо существуют замкнутые $F_{n} \subset U_{n}$ maкuе, что $\bigcup_{n=1}^{\infty} F_{n}=P$; (c) если $F_{n} \subset P$ замкнуты, $F_{n} \supset F_{n+1}, \quad n=1,2, \ldots, \bigcap_{n=1}^{\infty} F_{n}=\varnothing$, mо суиествугот открытые $G_{n} \supset F_{n}$ makue, чmo $\bigcap_{n=1}^{\infty} G_{n}=\varnothing$.

2. Назовем множества $X_{k} \subset P, k=1, \ldots, n$, отделцмыми в пространстве $P$, если существуют открытые $G_{k} \supset X_{k}$ такие, что $\bigcap_{k=1}^{n} \Psi_{k}=$ б.

2.1. Пусть $X_{\alpha} \subset U_{\alpha} \subset P, U_{\alpha}$ открыты, $\left\{U_{\alpha} ; \alpha \in A\right\}$ локально конечна. Существуют открытые $G_{\alpha}$ maкuе, что $X_{\alpha} \subset G_{a} \subset U_{\alpha} u \bigcap_{i=1}^{n} G_{a_{i}}=\varnothing$ всякий раз, когда $X_{a_{1}}, \ldots, X_{a_{n}}$ отделимы.

Доказательство. Достаточно показать, что из указанных предположений вытекает (при любом $\alpha_{0} \epsilon A$ ) существование открыного $G$ такого, что (1) $X_{a_{0}} \subset G \subset U_{a_{0}}$, (2) если $X_{a_{0}}, X_{a_{1}}, \ldots, X_{a_{n}}$ отделимы, то отделимы также $G, X_{a_{1}}, \ldots, X_{\alpha_{n}}$. Действительно, тогда мы сможем легко доказать 2.1, напр. на основании трансфинитной индукции (вполне упорядочив $A$ и постепенно заменяя $X_{\alpha}$ через $G_{\alpha}$ ). Обозначим $A^{*}$ множество $a \epsilon[A]$ таких, что $a_{0} \epsilon a$, множества $X_{a}, a \epsilon a$, отделимы в $P$. Для каждого $a \in A^{*}$ найдем открытые $V_{a}^{a} \supset X_{a}, a \in a$, такие, что $\bigcap_{a \in a} V_{a}^{a}=ø$; очевидно, можно еще потребовать $V_{a}^{a} \subset U_{\alpha}$. Для $a \in A^{*}$ положим $S_{a}=\bigcap_{a \in a-\left(a_{0}\right)} V_{a}^{a}$; пусть $S=\bigcup S_{a}$. Очевидно, $\bar{S}_{a} \cap X_{a_{0}}=\varnothing ;$ из 1.1 вытекает, что $\left\{S_{a}\right\}$ локально конечна, так что $\bar{S}=\bigcup \bar{S}_{a}$; итак, $\bar{S} \cap X_{n_{0}}=\varnothing$. Теперь достаточно положить $G=U_{\alpha_{0}}-\bar{S}$.

2.2. Теорема. Если $P$ нормально, $F_{a} \subset U_{a} \subset P, F_{a}$ замкнуты, $U_{a}$ открыты, $\left\{U_{a}\right\}$ лэкально кэнечнх, то сучестзуют открытые $G_{a}^{a}$ maкue, что $F_{\alpha} \subset G_{\alpha} \subset U_{\alpha},\left\{F_{a}\right\} \cong\left\{G_{\alpha}\right\}$.

Эта известная теорема (см. [9]) вытекает из 2.1 и того, что в нормальном $P$ замкнутые $F_{k}$ такие, что $\bigcap_{k=1}^{n} F_{k}=\varnothing$, отделимы.

2.3. Если $P$ наследственно нормально, $S \subset P, X_{k} \subset S, k=1, \ldots, n$, и $X_{1}, \ldots, X_{n}$ отделимы в $S$, то они отдёлимы в $P$.

Эта лемма хорошо известна; см. напр. [3], 1.16.

2.4. Теорема. Если $P$ наследственно нормально, $X_{\alpha} \subset U_{\alpha} \subset P, U_{\alpha}$ открыты в $P, X_{a}$ открытьы в $X=\bigcup X_{\alpha},\left\{U_{\alpha}\right\}$ локально конечна, mo существуют открытые $G_{\alpha} \subset U_{a}$ makue, что $X \cap G_{a}=X_{\alpha}$, $\left\{G_{a}\right\} \cong\left\{X_{a}\right\}$.

3. Система $\left\{X_{\alpha}\right\}, X_{\alpha} \subset P$, называется дискретной (в $P$ ), если она покально конечна и $\bar{X}_{\text {a }}$ попарно не пересекаются. Пространство $P$ называется коллективно нормальным, если для любой дискретной $\left\{X_{a}\right\}$ существуют открытые $G_{a} \supset X_{\alpha}$ такие, что $\left\{G_{a}\right\} \cong\left\{X_{a}\right\}$.

3.1. Для того, чтобы нормальное $P$ было коллективно нормальным, необходимо и достаточно каждое из следующих условий: (а) если $F_{\alpha} \subset P$ замкнуты, $\left\{F_{\alpha}\right\}$ локально конечна и имеет конечную кратность, то существуют открытые $G_{a} \supset F_{a}$ такие, что $\left\{G_{a}\right\}$ локально конечна; (b) если $S \subset P$ замкнцто, $\{F\}$ (соответственно, $\{$ н $\}$ ) является локально конечным замкнутымм (открытым) покрытием $S, F_{\alpha} \subset H_{\alpha}$, то существуют открытые $G_{\alpha}$ maкuе, что $F_{\alpha} \subset S \cap G_{a} \subset H_{\alpha},\left\{G_{a}\right\}$ локально конечна.

Доказательство. І. Пусть $P$ коллективно нормально. Докажем (a) индукцией по кратности $n$ системы $\left\{F_{\alpha} ; \alpha \in A\right\}$. Легко установить, что (а) верно для $n=1$. Пусть (а) доказано для $n \leqslant m$; пусть $\left\{F_{\alpha}\right\}$ имеет кратность $m+1$. Обозначим $A^{*}$ мнонество всех $a \subset A$, состо- 
ящих ровно из $m+1$ элементов. Тогда $\left\{\bigcap_{a \in a} F_{a} ; a \in A^{*}\right\}$ дискретна и потому существугот (так как (а) верно для $n=1$ ) открытые $U_{a} \supset \bigcap_{a \in a} F_{a}$ такие, что $\left\{U_{a} ; a \in A^{*}\right\}$ локально конечна; множества $U_{a}$ притом можно подобрать так, чтобы $U_{a} \cap F_{\alpha}=\varnothing$ при $\alpha$ non $\epsilon$. Положим $U=\bigcup U^{a}$. Тогда $\left\{F_{a}-U\right\}$ имеет кратность $\leqslant m$, так что существугот открытые $V_{a} \supset F_{a}-U$ такие, что $\left\{V_{a}\right\}$ локально конечна. Положим $G_{a}=V_{a} \cup \bigcup_{a \in a} U_{a}$. Легко установить, что $G_{a}$ имегот нужные свойства.

II. Донажем, что из (a) вытекает (b). Обозначим $S_{n}$ (соответственно, $T_{n}$ ) множество $x \in S$ принадлежащих не более чем $n$ множествам $F_{\alpha}$ (соответственно, $H_{a}$ ). Јегко видеть, что $S_{n}$ открыты в $S, T_{n}$ замкнуты, $\bigcup T_{n}=S$. Из 1.3 и 1.2 вытекает, что существует вписанное в $\left\{S_{n}\right\}$ счетное звездно конечное замкнутое покрытие $\left\{\Phi_{k}\right\}$ прострапства $S$. Из 1.4 вытекает, что существуют открытые (в $P$ ) $U_{l_{k}} \supset \Phi_{k}$ такие, что $\left\{U_{k}\right\}$ звездно конечна. Найдем ещз открытые $V_{k}$ такие, что $U_{k} \supset \bar{V}_{k}, \quad V_{k} \supset \Phi_{k} ;$ тогда $\left\{V_{k}\right\}$ локально конечна. Для $k=1,2, \ldots$ система $\left\{F_{a} \cap \Phi_{k}\right\}$ локально конечна и имеет гонечную кратность, так что существуют открытые $G_{a, k}^{*} \supset F_{a} \cap \Phi_{k}$ такие, что $\left\{G_{a, k}^{*}\right\}$ локально конечна. Теперь достаточно, взяв еще открытые $W_{\alpha}$ такие, что $S \frown W_{\alpha}=H_{\alpha}$, положить $G_{\alpha}=W_{\alpha} \frown \bigcup_{k}\left(G_{a, k}^{*} \cap V_{k}\right)$.

III. Из (b) вытекает на основании 2.2 , что $P$ коллективно нормально.

3.2. Теорема. Для того, чтобы нормальное $P$ было коллективно нормальным, необходимо и достаточно, чтобы для любого замкнутого SCP и его локально конечного открытого покрытия $\left\{X_{a}\right\}$ существовали открытые $G_{\alpha}$ maкuе, что $\bigcup G_{\alpha} \supset S, S \cap G_{\alpha} \subset X_{\alpha},\left\{G_{a}\right\}$ локально конечна.

Эта теорема, легко вытекающая из 3.1 (и 1.2), известна; см. [2].

3.3. Теорема. Если $P$ коллективно нормально, то для любого замкнутого SCP и его локально конечного открытого покрытия $\left\{X_{\alpha}\right\}$ существуют открытые $G_{a}$ maкuе, что $\bigcup G_{a} \supset S, G_{\alpha} \cap S \subset X_{\alpha},\left\{G_{\alpha}\right\}$ локально конечна, $\left\{G_{\alpha} \cap S\right\} \cong\left\{X_{\alpha}\right\} \cong\left\{G_{\alpha}\right\} \cong\left\{\bar{G}_{a}\right\}$.

Наоборот, если для $P$ выполняетсл это условие, то $P$ коллективно нормально.

Докавательство. Существование упомянутых $G_{\alpha}$ получим так, что сначала подберем (легко доказать, что это возможно) замкщутыте $F_{a} \subset X_{a}$ так, чтобы $\bigcup F_{a}=S,\left\{F_{a}\right\} \cong\left\{X_{a}\right\}$, применим 3.1, а затем 2.2.

4. Назовем пространство сильно нормальным, если оно коллективно нормально и счетно паракомпактно.
4.1. Теорема. Нормальное $P$ сильно нормально, если и только если для любой локально конечной $\left\{X_{\alpha}\right\}, X_{\alpha} \subset P$, существуют открытые $G_{a} \supset X_{a}$ mакие, что $\left\{G_{\alpha}\right\}$ локально конечна в $P$.

Доказательство. I. Если $P$ сильно нормально, положим $F_{\alpha}=\bar{X}_{a}, S=\bigcup F_{a}$, так что $S$ вамкнуто. Дальнейший ход доказательства аналогичен II части доказательства 3.1 с той разницей, что существование счетного звездно конечного замкнутого покрытия $\left\{\Phi_{k}\right\}$, вписанного в $\left\{S_{n}\right\}$, вытекает из 1.5(a) и 1.2 .

II. Если условие выполняется, то коллентивная нормальность вытекает из 3.1 , счетная паракомпактность из 1.5 (с), так как если $F_{n}$ замкнуты, $F_{n} \supset F_{n+1}, n=1,2, \ldots, \bigcap_{n=1}^{\infty} F_{n}=\emptyset$, то $\left\{F_{n}\right\}$ локально конечна и потому существуют открытые $G_{n} \supset F_{n}$ такие, что $\left\{G_{n}\right\}$ локально конечна, и, следовательно, $\bigcap_{n=1}^{\infty} G_{n}=\emptyset$.

4.2. Теорема. Если $P$ сильно нормально, то для любой локально конечной системы $\left\{F_{a}\right\}$ замкнутых множсеств существуют открытые $G_{\alpha} \supset F_{a}$ maкие, что $\left\{G_{a}\right\}$ локально конечна, $\left\{G_{a}\right\} \cong\left\{F_{a}\right\}$.

4.3. ТЕорема. Если $P$ сильно нормально, то для любого замкнутого SCP и его локально конечного открытого покрытия $\left\{X_{a}\right\}$ cyцествуют открытые $G_{a}$ такие, что $G_{a} \cap S=X_{\alpha},\left\{G_{a}\right\}$ локально конечна.

4.4. Теорема. Если $P$ сильно нормально и наследственно нормально, то для любого замкнутого $S \subset P$ и его локально конечного открытого покрытия $\left\{X_{\alpha}\right\}$ существуют открытые $G_{a}$ maкие, что $G_{a} \cap S=X_{a}$, $\left\{G_{a}\right\}$ локально конечна, $\left\{G_{a}\right\} \cong\left\{X_{a}\right\}$.

Это вытекает из 4.3 и 2.4 .

4.5. Замечание. Јегко установить, что из условия указанного в 4.3 вытекает, что $P$ ноллективно нормально, а из условия в 4.4 вытекает, что $P$ коллективно нормально и наследственно нормально. Автору не удалось найти ответа на следующие вопросы: $\mathbf{P} \mathbf{2 4 3}$ достаточно ли условие в 4.3 для сильной нормальности нормального $P$, $\mathrm{P}$ 244. необходимо ли оно для коллективной нормальности $P, \mathbf{P} 245$. дсстаточно ли условие в 4.4 для того, qтобы $P$ было сильно нормальным, $\mathrm{P}$ 246. необходимо ли оно для того, чтобы $P$ было коллективно нормальным и наследственно нормальным.

5. Пусть $X_{\alpha} \subset P$. Назовем систему $\left\{X_{\alpha}\right\}$ равномерно локально конечной (в $P$ ), если существует локально конечное открытое покрытие $\left\{U_{\beta}\right\}$ пространства $P$, относительно которого она конечна. 
5.1. Теорема. Пусть $X_{a} \subset P$. Дляя moro, чтобы $\left\{X_{a}\right\}$ была равномерно локально конечной, необходимо и достаточно, чтобы существовали открытые $G_{a} \supset \bar{X}_{\alpha}$ такие, что $\left\{G_{a}\right\}$ локально конечна.

Доказательство. I. Пусть $\left\{X_{a}\right\}$ равномерно локально коненна; пусть $\left\{U_{\beta}\right\}$ имеет указанные свойства. Обозначим $G_{\alpha}$ обьединепие всех $U_{\beta}$, пересекающих $X_{a}$; легко видеть, что $G_{\alpha}$ имеют нуяные свойства.

II. Пусть $G_{\alpha} \supset \bar{X}_{\alpha}$ открыты, $\left\{G_{\alpha} ; \alpha \in A\right\}$ локально конечна. Для $a \epsilon[A]$ положим $U_{a}=\bigcap_{a \in a} G_{a}-\bigcup_{\text {nопе } e a} \bar{X}_{a}$ (в частности, для $a=\varnothing$ имеем $\left.U_{a}=P-\bigcup \bar{X}_{a}\right)$. Легко видеть, что $\bigcup U_{a}=P$; из 1.1 вытекает, тто $\left\{U_{a}\right\}$ локально конечна; если $U_{a} \cap \bar{X}_{\alpha} \cong \varnothing$, то, очевидно, $\alpha \in \alpha$; итак, $\left\{U_{a}\right\}$ имеет нужные свойства.

5.2. Теорема. Если $P$ нормально, $\left\{X_{a}\right\}$ равномерно локально конечна в $P$, то существует равномерно локально конечное открытое покрытие $\left\{V_{\beta}\right\}$, относительно которого $\left\{X_{\alpha}\right\}$ конечна.

Доказательство. Взяв $U_{\beta}$ указанные в определении, найдем на основании 1.2 открытые $V_{\beta}$ такие, что $\bar{V}_{\beta} \subset U_{\beta}, \cup V_{\beta}=P$, и применим к ним 5.1 .

5.3. ТЕорема. Для того, чтобы нормальное $P$ было коллективно нормальным, необходим и достаточно каждое из следующих условий: (а) если система $\left\{X_{a}\right\}$ равномерно локально конечна в замкнутом SCP, то она равномерно локально конечна в $P$; (b) для лобого замкнутого SCP и его равномерно локального конечного открытого покрытия $\left\{X_{\alpha}\right\}$ существует равномерно локально конечное открытое покрытие $\left\{\hat{f}_{a}\right\}$ пространства $P$ makoе, чmo $G_{a} \cap S=X_{\alpha}$.

Доказательство. I. Докажем, что в коллективно нормальном $P$ имеет место $(\mathrm{b})$. Действительно, согласно 5.1 существугот открытые в $S$ множества $H_{a} \supset \bar{X}_{a}$ такие, что $\left\{H_{a}\right\}$ локально конечна; теперь применим 3.1 .

II. Из (b) вытекает (a) на основании 5.2.

III. Если имеет место (а), а система $\left\{F_{a}\right\}$ замкпутых множеств дискретна, то, очевидно, $\left\{F_{a}\right\}$ равномерно локально конеина в $S=\bigcup F_{a}$ и, следовательно, в $P$. Теперь прнменим 5.1, а затем 2.2.

5.4. Теорема. Для того, чтобы нормальное пространство было сильно нормальным, необходимо и достаточно, чтобы камсдая кокальо конечнал система его подмножества была равномерно локально конечной.

Эта теорема вытегает непосредственно из 4.1 и 5.1.

5.5. Теорема. Если $P$ коллективно нормально и наследственно нормально, то для любого замкнутого S С $P$ и его равномерно локально конечного покрытия $\left\{\dot{X}_{\alpha}\right\}$ сущестеуют открытые $G_{a}$ mакие, что $G_{\alpha} \cap S=X_{a},\left\{G_{a}\right\}$ равномерно локально конечна, $\left\{G_{a}\right\} \cong\left\{X_{a}\right\}$.

Это вытекает из 5.3 и 2.4 .

\section{ЦИТИРОВАННАЯ ЛИТЕРАТУРА}

[1] J. Dinudonnó, Une généralisation des espaces compacts, Journal dos Mathématiquos pures ot appliquéos (9) 23 (1944), crp. 65-76.

[2] C. H. Dowkor, On a theorem of Hanner, Arkiv för Matematik 2 (1952), стр. $307-313$.

[3] М. К а те то в, О размерности несепарабельныхх пространств I, Чехословацкий Математический Журнал 2 (77) (1052), стр. 333-368.

[4] J. L. Kelloy, General topology, 1955.
[5] C. Kuratowski, Sur le prolongement des fonctions continues et les transformations en polytopes, Fundamenta Mathematicae 24 (1935), стр. 259-268.

[6] - Topologie I, Warszawa-Wroclaw 1948.

[7] M. J. Mansfield, On countably paracompact spaces, Canadian Journal of Mathomatics 9 (1957), crp. 443-449.

[8] K. Murita, Star-finite coverings and star-finite property, Mathematica Japonicao 1 (1948), crp. 60-68.

[9] - On the dimension of normal spaces II, Journal of the Mathematical Socioty of Japan 2 (1950), crp. 16-23.

Reçu par la Rédaction le 30. 11. $195 \%$ 